\title{
Dampening neuroinflammation
}

\section{By Joanne Kotz, Senior Editor}

A California team has shown that monoacylglycerol lipase, which controls levels of a pain-reducing metabolite in the brain, also regulates neuroinflammation. ${ }^{1}$ The researchers have proof of concept that a small molecule inhibitor of the target blocks inflammation and decreases neurodegeneration in a mouse model of Parkinson's disease, and they are now studying the compound in additional neurodegenerative and neurological diseases.

Newco Abide Therapeutics is exercising an option to license the monoacylglycerol lipase (MAGL) inhibitors.

MAGL is a serine hydrolase enzyme that degrades 2-arachidonoylglycerol (2-AG), a ligand of pain-relieving cannabinoid receptors in the brain. In 2008,

"Targeting MAGL might provide a combined pain and anti-inflammatory mechanism that could be interesting not only for PD but also for many other neurological diseases." -Johan Luthman, Merck \& Co. Inc. a team led by Benjamin Cravatt identified a brain-permeable small molecule inhibitor of MAGL that causes $2-A G$ levels to increase in the brain, inducing analgesia in mice. ${ }^{2}$

Cravatt is chair of the Department of Chemical Physiology at The Scripps Research Institute.

At the time, Cravatt and collaborators observed that in addition to boosting 2-AG levels, MAGL inhibitors also triggered a decrease in brain levels of arachidonic acid, the precursor molecule to inflammatory prostaglandins. ${ }^{2,3}$ Phospholipase enzymes had previously been considered the principle regulators of arachidonic acid levels, and these observations suggested a link between MAGL activity and prostaglandin-mediated neuroinflammation.

Based on this, a team led by Cravatt and Daniel Nomura, an assistant professor in the Department of Nutritional Science and Toxicology at the University of California, Berkeley, has now looked more closely at the impact of blocking MAGL on brain inflammation.

In mice stimulated with lipopolysaccharide (LPS) to induce neuroinflammation, Magl deficiency or a MAGL inhibitor prevented increases in inflammatory prostaglandins and cytokines and blocked microglial activation compared with Magl expression or vehicle (see Figure 1, "Connecting cannabinoid and prostaglandin pathways").

Moreover, blocking Magl reduced LPS-stimulated increases in brain prostaglandin levels at least three-fold, whereas blocking
cPla 2 - $\alpha$ (phospholipase $\mathrm{A}_{2}$ group IVA cytosolic calcium-dependent; $\mathrm{Pla}_{2} \mathrm{~g} 4 \mathrm{a}$ ), which is a known regulator of prostaglandin production in neuroinflammation, only resulted in an approximately $20 \%$ decrease in LPS-induced prostaglandins.

In a mouse model of neurotoxin-induced Parkinson's disease (PD), the MAGL inhibitor reduced neuroinflammation and dopaminergic neurodegeneration compared with vehicle.

Finally, the team asked whether MAGL also controlled inflammation in peripheral tissues. In LPS-treated mice, Magl regulated prostaglandin levels in the liver and lung, whereas $\mathrm{cPla}_{2}-\alpha$ controlled levels in the gut and spleen. Neither Magl nor $\mathrm{cPla}_{2}-\alpha$ regulated prostaglandin production in the heart and kidney.

Results were published in Science.

"It's been thought since the early 1900s that phospholipases dominate the production of arachidonic acid," said Nomura. It now appears there are tissue-specific mechanisms that control production, he added.

\section{Casting a MAGL net}

The work "opens up another angle to approach inflammation in the brain," said Johan Luthman, leader of the Early Development

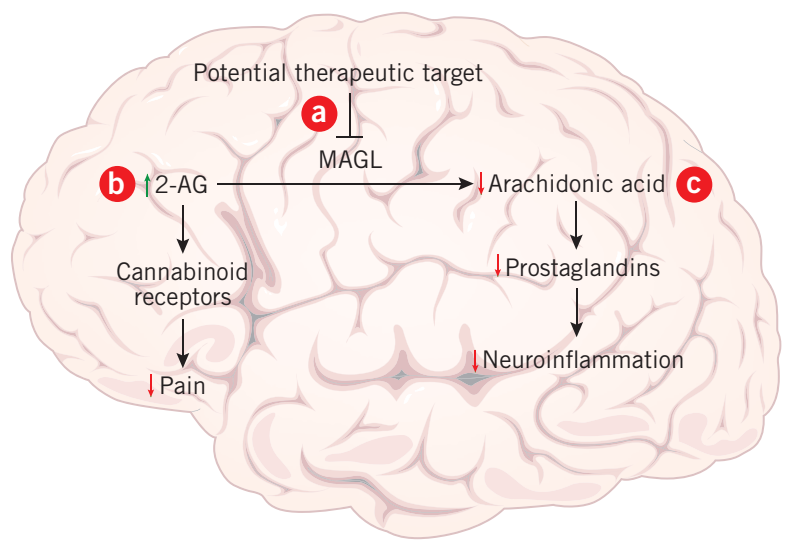

Figure 1. Connecting cannabinoid and prostaglandin pathways. A team led by researchers from The Scripps Research Institute and the University of California, Berkeley have shown that monoacylglycerol lipase (MAGL), which controls levels of a pain-reducing metabolite in the brain, also regulates neuroinflammation. Thus, blocking MAGL could provide a therapeutic benefit in neurodegenerative disorders characterized by neuroinflammation.

Previous work from Scripps had shown that in mice, a small molecule inhibitor of MAGL [a] increased the levels of 2-arachidonoylglycerol (2-AG) in the brain, which acted through the cannabinoid receptors to decrease pain $[\mathbf{b}]$. The team has now shown that in a mouse model of neuroinflammation, inhibiting MAGL lowers the production of arachidonic acid to decrease levels of inflammatory prostaglandins and blunt inflammation in the brain [c].

In a mouse model of neurotoxin-induced Parkinson's disease (PD), a MAGL inhibitor reduced neuroinflammation and dopaminergic neurodegeneration compared with vehicle. 
Neuroscience and Ophthalmology Franchise at Merck \& Co. Inc. "Targeting MAGL might provide a combined pain and anti-inflammatory mechanism that could be interesting not only for PD but also for many other neurological diseases."

The weakness of the paper is the use of an acute, neurotoxin-based PD model, said Luthman. He noted that results from this model have not translated well into the clinic and suggested the authors should test MAGL inhibitors in newer PD

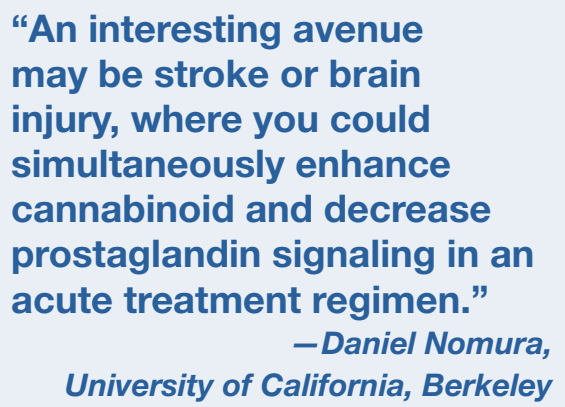

"An interesting avenue may be stroke or brain injury, where you could simultaneously enhance cannabinoid and decrease prostaglandin signaling in an acute treatment regimen."

-Daniel Nomura,

University of California, Berkeley

simultaneously enhance cannabinoid and decrease prostaglandin signaling in an acute treatment regimen."

Given MAGL's involvement in liver and lung inflammation, Nomura said inhibitors might also have a therapeutic benefit in fibrosis of those tissues.

Scripps holds patents on the MAGL inhibitor used in the Science paper. Abide Therapeutics, which was cofounded in 2011 by Cravatt to develop serine hydrolase inhibitors, is exercising its exclusive option to license patents covering MAGL inhibitors, according to CEO Alan Ezekowitz. "The MAGL program is the most advanced program, and the company intends to explore MAGL inhibitors in the treatment of pain, neuroinflammation and potentially neurodegenerative diseases like Parkinson's disease," said Ezekowitz.

Kotz, J. SciBX 4(45); doi:10.1038/scibx.2011.1254

Published online Nov. 17, 2011

\section{REFERENCES}

1. Nomura, D.K. et al. Science; published online Oct. 20, 2011; doi:10.1126/science.1209200

Contact: Benjamin F. Cravatt, The Scripps Research Institute, La Jolla, Calif.

e-mail: cravatt@scripps.edu

Contact: Daniel K. Nomura, University of California, Berkeley, Calif. e-mail: dnomura@berkeley.edu

2. Long, J.Z. et al. Nat. Chem. Biol. 5, 37-44 (2009)

3. Nomura, D.K. et al. Nat. Chem. Biol. 4, 373-378 (2008)

COMPANIES AND INSTITUTIONS MENTIONED

Abide Therapeutics, La Jolla, Calif.

Merck \& Co. Inc. (NYSE:MRK), Whitehouse Station, N.J.

The Scripps Research Institute, La Jolla, Calif.

University of California, Berkeley, Calif. 